\title{
Unpredictability in Economic Analysis, Econometric Modeling and Forecasting
}

\author{
David F. Hendry \\ Department of Economics, and Institute for Economic Modelling, \\ Oxford Martin School, University of Oxford, UK. \\ Grayham E. Mizon \\ Faculty of Social and Human Sciences, University of Southampton \\ and Institute for Economic Modelling, Oxford Martin School, University of Oxford, UK. *
}

\begin{abstract}
Unpredictability arises from intrinsic stochastic variation, unexpected instances of outliers, and unanticipated extrinsic shifts of distributions. We analyze their properties, relationships, and different effects on the three arenas in the title, which suggests considering three associated information sets. The implications of unanticipated shifts for forecasting, economic analyses of efficient markets, conditional expectations, and inter-temporal derivations are described. The potential success of general-to-specific model selection in tackling location shifts by impulse-indicator saturation is contrasted with the major difficulties confronting forecasting.
\end{abstract}

JEL classifications: C51, C22.

Keywords: Unpredictability; 'Black Swans'; Distributional shifts; Forecast failure; Model selection; Conditional expectations.

\section{Introduction}

Unpredictability has been formalized as intrinsic stochastic variation in a known distribution, where conditioning on available information does not alter the outcome from the unconditional distribution, as in the well-known prediction decomposition, or sequential factorization, of a density (see Doob, 1953). Such variation can be attributed (inter alia) to chance distribution sampling, 'random errors', incomplete information, or in economics, many small changes in the choices by individual agents. A variable that is intrinsically unpredictable cannot be modeled or forecast better than its unconditional distribution.

However, the converse does not hold: a variable that is not intrinsically unpredictable may still be essentially unpredictable because of two additional aspects of unpredictability. The first concerns independent draws from fat-tailed or heavy-tailed distributions, which leads to a notion we call 'instance

* This research was supported in part by grants from the Open Society Foundations and the Oxford Martin School. We are indebted to Gunnar Bärdsen, Jennifer L. Castle, Neil R. Ericsson, Søren Johansen, Bent Nielsen, Ragnar Nymoen, Felix Pretis, Norman Swanson and two anonymous referees for helpful comments on earlier versions. Forthcoming, Journal of Econometrics. Contact details: david.hendry@nuffield.ox.ac.uk and grayham.mizon@soton.ac.uk. 
unpredictability'. Here the distribution of a variable that is not intrinsically unpredictable is known, as are all conditional and unconditional probabilities, but there is a non-negligible probability of a very discrepant outcome. While that probability is known, it is not known on which draw the discrepant outcome will occur, nor its magnitude, leading to a 'Black Swan' (as in Taleb, 2007), with potentially large costs when that occurs (see Barro, 2009). The third aspect we call 'extrinsic unpredictability', which derives from unanticipated shifts of the distribution itself at unanticipated times, of which location shifts (changes in the means of distributions) are usually the most pernicious. Intrinsic and instance unpredictability are close to 'known unknowns' in that the probabilities of various outcomes can be correctly pre-calculated, as in rolling dice, whereas extrinsic unpredictability is more like 'unknown unknowns' in that the conditional and unconditional probabilities of outcomes cannot be accurately calculated in advance (as in the first quote of Clements and Hendry, 1998). The recent financial crisis and ensuing deep recession have brought both instance and extrinsic unpredictability into more salient focus (see Taleb, 2009, and Soros, 2008, 2010, respectively).

These three aspects of unpredictability suggest that different information sets might explain at least a part of their otherwise unaccounted variation. This is well established both theoretically and empirically for intrinsic unpredictability, where 'regular' explanatory variables are sought. Empirically, population distributions are never known, so even to calculate the probabilities for instance unpredictability, it will always be necessary to estimate the distributional form from available evidence, albeit few 'tail draws' will occur from which to do so. New aspects of distributions have to be estimated when extrinsic unpredictability occurs. Consequently, each type of unpredictability has substantively different implications for economic analyses, econometric modeling, and economic forecasting. Specifically, inter-temporal economic theory, forecasting, and policy analyses could go awry facing extrinsic unpredictability, yet $e x$ post, the outcomes that eventuated are susceptible to being modeled. We briefly discuss the possible role of impulse-indicator saturation for detecting and removing in-sample location shifts. The availability of such tools highlights the contrast between the possibilities of modeling extrinsic unpredictability ex post against the difficulties confronting successful ex ante forecasting, where one must forecast outliers or shifts, which are demanding tasks. However, transformations of structural models that make them robust after shifts, mitigating systematic forecast failure, are feasible.

The structure of the paper is as follows. Section 2 considers intrinsic unpredictability in $\S 2.1$; instance unpredictability in $\S 2.2$; and extrinsic unpredictability in $\S 2.3$. Theoretical implications are drawn in section 3, with the relationships between intrinsic, instance and extrinsic unpredictability in $\S 3.1$, and the impact of reduced information in $§ 3.2$. The possibility of three distinct information sets associated 
respectively with 'normal causality', the timing of outliers, and the occurrence of distributional shifts is discussed in $§ 3.3$. The difficulties both economists and economic agents confront facing unanticipated breaks are analyzed in $\$ 3.4$. Section 4 investigates some consequences for empirical applications. The fundamental separation between modeling and forecasting from instance and extrinsic unpredictabilitybut not intrinsic unpredictability-is discussed in $\S 4.1$. Then $\S 4.2$ considers the relationships between the three aspects of unpredictability for model selection in processes with unanticipated breaks, leading to a reconsideration of the role of congruent modeling for forecasting in $\S 4.3$. These analyses are illustrated in $\S 4.4$ by an empirical application of robust forecasting. Section 5 concludes.

\section{Unpredictability}

We now consider the three distinct sources of unpredictability. Were it the case that the data generation process (DGP) changed unexpectedly at almost every data point, then reliable inferences would be rendered essentially impossible. Fortunately, the various sources of unpredictability are less extreme than this, so inference remains possible subject to the caveats discussed in the following.

\subsection{Intrinsic unpredictability}

Definition 1 A non-degenerate n-dimensional vector random variable $\epsilon_{t}$ is an intrinsically unpredictable process with respect to an information set $\mathcal{I}_{t-1}$, which always includes the sigma-field generated by the past of $\boldsymbol{\epsilon}_{t}$, denoted $\sigma\left[\mathbf{E}_{t-1}\right]$, over a time period $\mathcal{T}$ if the conditional distribution $\mathrm{D}_{\boldsymbol{\epsilon}_{t}}\left(\boldsymbol{\epsilon}_{t} \mid \mathcal{I}_{t-1}\right)$ equals the unconditional distribution $\mathrm{D}_{\epsilon_{t}}\left(\boldsymbol{\epsilon}_{t}\right)$ :

$$
\mathrm{D}_{\epsilon_{t}}\left(\epsilon_{t} \mid \mathcal{I}_{t-1}\right)=\mathrm{D}_{\epsilon_{t}}\left(\epsilon_{t}\right) \quad \forall t \in \mathcal{T}
$$

Intrinsic unpredictability is so-called as it is an intrinsic property of $\epsilon_{t}$ in relation to $\mathcal{I}_{t-1}$, not dependent on knowledge about $\mathrm{D}_{\epsilon_{t}}(\cdot)$, so is tantamount to independence between $\epsilon_{t}$ and $\mathcal{I}_{t-1}$. When $\mathcal{I}_{t-1}=\sigma\left[\mathbf{X}_{t-1}\right]$ (say) is the 'universal' information set, (1) clarifies why $\boldsymbol{\epsilon}_{t}$ is intrinsically unpredictable. Intrinsic unpredictability applies equally to explaining the past (i.e., modeling $\left\{\boldsymbol{\epsilon}_{t}, t=1, \ldots, T\right\}$ ) and forecasting the future from $T$ (i.e., of $\left\{\boldsymbol{\epsilon}_{t}, t=T+1, \ldots, T+h\right\}$ ): the best that can be achieved in both settings is the unconditional distribution, and $\mathcal{I}_{t-1}$ is of no help in reducing either uncertainty.

Expectations formed at time $t$ using a distribution $\mathrm{f}_{t}$ are denoted $\mathrm{E}_{\mathrm{f}_{t}}[\cdot]$, and the variance is denoted $\vee_{\mathrm{f}_{t}}[\cdot]$ for each point in $\mathcal{T}$. 
Theorem 1 When the relevant moments exist, intrinsic unpredictability in distribution entails unpredictability in mean and variance:

$$
\mathrm{E}_{\mathrm{f}_{t}}\left[\boldsymbol{\epsilon}_{t} \mid \mathcal{I}_{t-1}\right]=\mathrm{E}_{\mathrm{f}_{t}}\left[\boldsymbol{\epsilon}_{t}\right] \text { and } \mathrm{V}_{\mathrm{f}_{t}}\left[\boldsymbol{\epsilon}_{t} \mid \mathcal{I}_{t-1}\right]=\mathrm{V}_{\mathrm{f}_{t}}\left[\boldsymbol{\epsilon}_{t}\right]
$$

However, neither the former nor the latter alone need imply the other. As a well known example, $\boldsymbol{\epsilon}_{t} \sim$ $\mathrm{IN}_{n}\left[\boldsymbol{\mu}, \boldsymbol{\Omega}_{\epsilon}\right]$, denoting an independently distributed Gaussian variable with expected value $\mathrm{E}_{\mathrm{f}_{t}}\left[\boldsymbol{\epsilon}_{t}\right]=\boldsymbol{\mu}$ and variance $\mathrm{V}_{\mathrm{f}_{t}}\left[\boldsymbol{\epsilon}_{t}\right]=\boldsymbol{\Omega}_{\epsilon}$, is an intrinsically unpredictable process.

Intrinsic unpredictability is only invariant under non-singular contemporaneous transformations, as inter-temporal transforms must affect (1), implying that no unique measure of forecast accuracy exists (see e.g., Leitch and Tanner, 1991, Clements and Hendry, 2005, and Granger and Pesaran, 2000a, 2000b). Thus, predictability requires combinations with $\mathcal{I}_{t-1}$, as in, for example:

$$
\mathbf{y}_{t}=\boldsymbol{\psi}_{t}\left(\mathbf{X}_{t-1}\right)+\boldsymbol{\epsilon}_{t} \text { where } \boldsymbol{\epsilon}_{t} \sim \mathrm{ID}_{n}\left[\mathbf{0}, \boldsymbol{\Omega}_{\boldsymbol{\epsilon}}\right]
$$

so $\mathbf{y}_{t}$ depends on both the information set and the innovation component. Then:

$$
\mathrm{D}_{\mathrm{y}_{t}}\left(\mathbf{y}_{t} \mid \mathcal{I}_{t-1}\right) \neq \mathrm{D}_{\mathrm{y}_{t}}\left(\mathbf{y}_{t}\right) \quad \forall t \in \mathcal{T}
$$

In (3), $\mathbf{y}_{t}$ is predictable in mean even if $\epsilon_{t}$ is unpredictable as:

$$
\mathrm{E}_{\mathrm{D}_{\mathrm{y}_{t}}}\left[\mathbf{y}_{t} \mid \mathcal{I}_{t-1}\right]=\boldsymbol{\psi}_{t}\left(\mathbf{X}_{t-1}\right) \neq \mathrm{E}_{\mathrm{D}_{\mathrm{y}_{t}}}\left[\mathbf{y}_{t}\right]
$$

in general. Since:

$$
\mathrm{V}_{\mathrm{D}_{y_{t}}}\left[\mathbf{y}_{t} \mid \mathcal{I}_{t-1}\right]<\mathrm{V}_{\mathrm{D}_{\mathrm{y}_{t}}}\left[\mathbf{y}_{t}\right] \text { when } \mathrm{V}_{t}\left[\boldsymbol{\psi}_{t}\left(\mathbf{X}_{t-1}\right)\right] \neq \mathbf{0}
$$

predictability ensures a variance reduction, consistent with its nomenclature, since unpredictability entails equality in (5), and the 'smaller' the conditional variance matrix, the less uncertain is the prediction of $\mathbf{y}_{t}$ from $\mathcal{I}_{t-1}$.

\subsection{Instance unpredictability}

Definition 2 The vector random variable $\boldsymbol{\epsilon}_{t}$ is an instance unpredictable process over a time period $\mathcal{T}$ if there is a non-negligible probability of 'extreme draws' of unknown magnitudes and timings. 
As Taleb $(2007,2009)$ has stressed, rare large-magnitude events, or 'Black Swans', do unexpectedly occur. One formulation of that insight is to interpret 'Black Swans' as highly discrepant draws from fattailed distributions, where there is a constant, but small, probability of such an occurrence each period. Both the timing and the magnitude of the discrepant events are then unpredictable, even when the form of the distribution is known and constant over time. When a large outcome materializes unexpectedly at time $\tau$, say, substantial costs or benefits can result, sometimes both, but for different groups of agents. Barro (2009) estimates very high costs from 'consumption disasters', finding 84 events over approximately the last 150 years with falls of more than $10 \%$ per capita, cumulating to a total duration of almost 300 'bad' years across his sample of 21 countries, mainly due to wars. However, there is a marked reduction in their frequency after World War II.

Recent research on many financial variables has revealed a proliferation of 'jumps', as measured by bipower variation (see e.g., Barndorff-Nielsen and Shephard, 2004, 2006). These seem to be ex ante instance unpredictable events, superimposed on the underlying Ornstein-Uhlenbeck processes. To mitigate possibly large costs from financial outliers, Taleb (2009) argues for more robust systems that avoid dependence on the non-occurrence of very large draws: for example, systems with more nodes and less interdependence.

Empirically, the distributions of extreme future events cannot be known: tail properties are especially difficult to estimate from available data, and distributions may also shift over time. If 'Black Swans' are indeed genuinely independent large draws from fat-tailed distributions, no information could reveal their timings or magnitudes, so success in forecasting such draws is most unlikely without a crystal ball. However, such draws may not be independent of all possible information, so may be partially open to anticipation in some instances. A 'Black Swan' is often viewed as a large deviation in a differenced variable, as in asset market returns or price changes. If not rapidly reversed, such a jump, or collapse, entails a location shift in the corresponding level. Thus, instance unpredictability in the differences of variables entails extrinsic unpredictability in the levels (and vice versa), the topic to which we now turn.

\subsection{Extrinsic unpredictability}

Definition 3 The vector random variable $\epsilon_{t}$ is an extrinsically unpredictable process over a time period $\mathcal{T}$ if there are intrinsically unpredictable shifts in its distribution:

$$
\mathrm{D}_{\epsilon_{t+1}}(\cdot) \neq \mathrm{D}_{\epsilon_{t}}(\cdot) \text { for some } t \in \mathcal{T} \text {. }
$$


The key feature of extrinsic unpredictability is that the distributional shift is unanticipated, even for variables that would be partly predictable in the absence of a shift, as in:

$$
\mathrm{D}_{\mathrm{y}_{t+1}}\left(\mathbf{y}_{t} \mid \cdot\right) \neq \mathrm{D}_{\mathrm{y}_{t}}\left(\mathbf{y}_{t} \mid \cdot\right) \quad \forall t \in \mathcal{T}
$$

An important difference between instance unpredictability and extrinsic unpredictability arises under independent sampling. In the former, a 'Black Swan' relative to the usual outcomes is unlikely to also occur on the next draw, and even less likely in several successive outcomes. For example, there are potentially extreme draws from a Student's $t_{3}$, but those should occur rarely, and would be equally likely in either tail, although that would not be true for, say, a log-normal or some other extreme-value distributions. In general, from Chebyshev's inequality, when $\mathrm{E}[y]=\mu<\infty$ and $\mathrm{E}\left[(y-\mu)^{2}\right]=\sigma^{2}<\infty$ with $\sigma>0$, for any real number $s>0$ :

$$
\operatorname{Pr}(|y-\mu| \geq s \sigma) \leq \frac{1}{s^{2}}
$$

Thus, two successive ' $10 \sigma$ ' draws have a probability of less than one in 10,000: flocks of 'Black Swans' are improbable. ${ }^{1}$

However, when the mean of a distribution changes, as in some of the examples cited below, as well as in Barro (2009), successive outcomes are likely to be around the new mean, so a cluster appears. Although the first outcome after a mean shift would initially appear to be a 'Black Swan', even with independent draws it would be followed by many more outcomes that were discrepant relative to the original distribution, but not relative to its mean-shifted replacement.

Empirically, there have been many major shifts since 1400 , or indeed any epoch longer than 50 years, in demography (average age of death changing from around 40 to around 80, with the average number of births per female falling dramatically), real outcomes such as incomes per capita (increasing 6-8 fold), and all nominal variables (some 1000 fold since 1850). Current outcomes in the Western world are not highly discrepant draws from the distributions relevant in the Middle Ages, but 'normal' draws from distributions with very different means. Such shifts can be precipitated by changes in legislation, advances in science, technology and medicine, financial innovation, climatic and geological shifts, or political and economic regimes, among other sources. While these examples are of major shifts over long periods, the recent financial crisis, and the many similar examples in the last quarter of the 20th Century,

\footnotetext{
${ }^{1}$ This only applies to draws from a given distribution. As Gillian Tett, Financial Times Magazine, March 26/27, p54, remarks 'Indeed, black swans have suddenly arrived in such a flock...', as can happen when considering many distributions of economic, political, natural and environmental phenomena.
} 
demonstrate that sudden large shifts occur (see the cases documented by Barrell, Dury, Holland, Pain and te Velde, 1998, and Barrell, 2001, inter alia). In asset markets, endogenous changes in agents' behavior can alter the underlying 'reality', as argued by Soros $(2008,2010)$ in his concept of reflexivity, inducing feedbacks that can lead to dramatic changes as agents' views move together, changing the system, which thereby ends in a different state.

Moreover, the distributions of the differences of many economic variables have also changed radically, and are also not stationary. For example, real growth per capita was a fraction of one percent per annum till the Industrial Revolution (see Apostolides, Broadberry, Campbell, Overton and van Leeuwen, 2008, for the evidence, and Allen, 2009, for an insightful analysis of the Industrial Revolution), remained low for the next couple of hundred years, but is now around 2\% pa in OECD countries, and much higher in emerging economies borrowing modern Western technology.

Shifts of distributions remain common, and would be unproblematic per se if they could be modeled and predicted. Co-breaking, where location shifts cancel, would enable some aspects to be forecast even when breaks themselves could not (see Hendry and Massmann, 2007), analogously to cointegration reducing stochastic trends in some I(1) variables to stationarity. Differencing plays a similar role, removing unit roots and converting location shifts to impulses. Many of the examples of demographic shifts noted earlier have such a property: annual changes in average age of death in OECD countries have been remarkably constant at about a weekend a week since around 1860, other than a major temporary shift during the First World War and the ensuing flu' epidemic. However, the distributions of changes in many economic variables also shift unexpectedly, especially distributions of nominal variables, but the change in the change may be more constant as few variables permanently accelerate (e.g., as with 'inflation surprises', where $\Delta^{2} p_{t}$, say, is sometimes treated as intrinsically unpredictable).

\section{Theoretical implications}

We now consider the theoretical implications of, and links between, the three sources of unpredictability, and in section 4 , discuss their practical consequences. 


\subsection{Intrinsic, instance and extrinsic unpredictability}

When (3) holds, $\mathbf{y}_{T+1}$ is not intrinsically unpredictable, but there are four reasons why $\mathbf{y}_{T+1}$ may not be usefully predicted from time $T$ using an estimated version of:

$$
\widehat{\mathbf{y}}_{T+1 \mid T}=\boldsymbol{\psi}_{T+1}\left(\mathbf{X}_{T}\right)
$$

The first is that in practice, (9) is never available, so instead forecasters must use:

$$
\widetilde{\mathbf{y}}_{T+1 \mid T}=\widetilde{\boldsymbol{\psi}}_{T+1}\left(\mathbf{X}_{T}\right)
$$

where $\widetilde{\boldsymbol{\psi}}_{T+1}(\cdot)$ is a forecast of what $\boldsymbol{\psi}_{T+1}(\cdot)$ will be. The second is that $\mathbf{X}_{T}$ may not be known at $T$, or may be incorrectly measured by a flash or nowcast estimate.

The third reason is instance unpredictability, which arises when the draw of $\epsilon_{T+1}$ in (3) induces outcomes $\mathbf{y}_{T+1}$ that are far from the forecast $\widehat{\mathbf{y}}_{T+1 \mid T}$ in the metric of $\boldsymbol{\Omega}_{\boldsymbol{\epsilon}}$, so that:

$$
\widehat{\boldsymbol{\epsilon}}_{T+1 \mid T}=\mathbf{y}_{T+1}-\widehat{\mathbf{y}}_{T+1 \mid T}
$$

is unexpectedly large (as in Taleb, 2009). That problem can occur even when $\boldsymbol{\psi}_{T+1}(\cdot)$ is known.

The fourth reason is that the distribution shifts in unanticipated ways at unexpected time points:

$$
\mathrm{D}_{\mathrm{y}_{T+1}}(\cdot) \neq \mathrm{D}_{\mathrm{y}_{T}}(\cdot)
$$

Thus, even if $\mathbf{y}_{T+1}$ was predictable according to (4) when $\mathbf{X}_{T}$ was known at time $T$, the lack of knowledge of $\boldsymbol{\psi}_{T+1}(\cdot)$ in (9), or more realistically, of an accurate value $\widetilde{\boldsymbol{\psi}}_{T+1}(\cdot)$, makes $\mathbf{y}_{T+1}$ extrinsically unpredictable. That problem will be exacerbated by any in-sample non-constancy of the distribution making empirical modeling difficult. To successfully forecast from (10) not only entails accurate data on $\mathbf{X}_{T}$, but also requires both a 'normal' draw $\boldsymbol{\epsilon}_{T+1}$ (or forecasting the outliers) and that $\widetilde{\boldsymbol{\psi}}_{T+1}(\cdot)$ be close to $\psi_{T+1}(\cdot)$ even though shifts occur, together essentially needing a crystal ball. A process is doubly unpredictable when it is both intrinsically and extrinsically unpredictable, so the pre-existing unconditional distribution does not match that holding in the next period. For example, $\boldsymbol{\epsilon}_{t} \sim \mathrm{IN}_{n}\left[\boldsymbol{\mu}_{t}, \boldsymbol{\Omega}_{\boldsymbol{\epsilon}}\right]$ will be less predictable than expected from probabilities calculated using $\boldsymbol{\Omega}_{\epsilon}$ when future changes in $\boldsymbol{\mu}_{t}$ cannot be determined in advance. Location shifts induce systematic forecast failure, so will be specifically considered below. 
Nevertheless, there may exist additional information sets, denoted $\mathcal{L}_{T}$ and $\mathcal{K}_{T}$, which could respectively help predict the outliers or the shifts in (11), as discussed in section 3.3. Importantly, once a shift has happened, it may be explicable (at worst by indicator variables), so there is a potentially major difference between modeling and forecasting when (11) holds, an aspect addressed in section 4.2. First, we use the preceding analyses to resolve an apparent paradox from Clements and Hendry (2005), namely that the costs of using less information are small, whereas there can be large costs from not having information about shifts.

\subsection{Prediction from a reduced information set}

Theorem 4 Predictions remain unbiased, although less accurate, when using a subset of information, $\mathcal{J}_{t-1} \subset \mathcal{I}_{t-1}$ where $\mathcal{J}_{t-1}=\sigma\left[\mathbf{Z}_{t-1}\right]$.

Proof. When the DGP is (3), since $\mathrm{E}_{\mathrm{D}_{\mathbf{y}_{t-1}}}\left[\boldsymbol{\epsilon}_{t} \mid \mathcal{I}_{t-1}\right]=\mathbf{0}$, so $\left\{\boldsymbol{\epsilon}_{t}\right\}$ is unpredictable given all the information, it must be unpredictable from a subset so that:

$$
\mathrm{E}_{\mathrm{D}_{\mathbf{y}_{t-1}}}\left[\boldsymbol{\epsilon}_{t} \mid \mathcal{J}_{t-1}\right]=\mathbf{0}
$$

From (3):

$$
\mathrm{E}_{\mathrm{D}_{\mathbf{y}_{t-1}}}\left[\mathbf{y}_{t} \mid \mathcal{J}_{t-1}\right]=\mathrm{E}_{\mathrm{D}_{\mathbf{y}_{t-1}}}\left[\boldsymbol{\psi}_{t}\left(\mathcal{I}_{t-1}\right) \mid \mathcal{J}_{t-1}\right]=\phi_{t-1}\left(\mathbf{Z}_{t-1}\right)
$$

say. Letting $\mathbf{e}_{t}=\mathbf{y}_{t}-\phi_{t-1}\left(\mathbf{Z}_{t-1}\right)$ be the unexplained component from (13), then:

$$
\mathrm{E}_{\mathrm{D}_{\mathbf{y}_{t-1}}}\left[\mathbf{e}_{t} \mid \mathcal{J}_{t-1}\right]=\mathrm{E}_{\mathrm{D}_{\mathbf{y}_{t-1}}}\left[\mathbf{y}_{t} \mid \mathcal{J}_{t-1}\right]-\phi_{t-1}\left(\mathbf{Z}_{t-1}\right)=\mathbf{0}
$$

so $\mathbf{e}_{t}$ remains a mean innovation with respect to $\mathcal{J}_{t-1}$ when $D_{\mathbf{y}_{t-1}}$ is used.

However, since:

$$
\mathbf{e}_{t}=\epsilon_{t}+\boldsymbol{\psi}_{t}\left(\mathbf{X}_{t-1}\right)-\phi_{t-1}\left(\mathbf{Z}_{t-1}\right)
$$

taking expectations with respect to the complete information set $\mathcal{I}_{t-1}$ :

$$
\mathrm{E}_{\mathrm{D}_{\mathbf{y}_{t-1}}}\left[\mathbf{e}_{t} \mid \mathcal{I}_{t-1}\right]=\boldsymbol{\psi}_{t}\left(\mathbf{X}_{t-1}\right)-\mathrm{E}_{\mathrm{D}_{\mathbf{y}_{t-1}}}\left[\boldsymbol{\phi}_{t-1}\left(\mathcal{J}_{t-1}\right) \mid \mathcal{I}_{t-1}\right]=\boldsymbol{\psi}_{t}\left(\mathbf{X}_{t-1}\right)-\boldsymbol{\phi}_{t-1}\left(\mathbf{Z}_{t-1}\right) \neq \mathbf{0}
$$

Thus, $\mathbf{e}_{t}$ is not an innovation relative to $\mathcal{I}_{t-1}$ so from (15) and (16):

$$
\mathrm{V}_{\mathrm{D}_{\mathbf{y}_{t-1}}}\left[\mathbf{e}_{t}\right]=\mathrm{V}_{\mathrm{D}_{\mathbf{y}_{t-1}}}\left[\boldsymbol{\epsilon}_{t}\right]+\mathrm{V}_{\mathrm{D}_{\mathbf{y}_{t-1}}}\left[\boldsymbol{\psi}_{t}\left(\mathbf{X}_{t-1}\right)-\boldsymbol{\phi}_{t-1}\left(\mathbf{Z}_{t-1}\right)\right] \geq \mathrm{V}_{\mathrm{D}_{\mathbf{y}_{t-1}}}\left[\boldsymbol{\epsilon}_{t}\right]
$$


so larger variance predictions will usually result, again consistent with the concept of predictability.

Thus, in the context of intrinsic unpredictability, more relevant information improves the accuracy of prediction, but less information by itself does not lead to biased outcomes relative to $D_{y_{t-1}}$. Such a result conflicts with the intuition that a loss of information about what causes shifts can lead to badly biased forecasts. The resolution of this apparent paradox lies in the assumption in equations like (12), that $\mathrm{D}_{\mathbf{y}_{t-1}}$ is the relevant distribution for the calculations, which it is not when location shifts occur. As $\mathbf{y}_{t}=\boldsymbol{\psi}_{t}\left(\mathbf{X}_{t-1}\right)+\boldsymbol{\epsilon}_{t}$ was generated by $\mathrm{D}_{\mathbf{y}_{t}}(\cdot)$, that must embody any distributional shift, and hence:

$$
\mathrm{E}_{\mathrm{D}_{\mathbf{y}}}\left[\boldsymbol{\psi}_{t}\left(\mathcal{I}_{t-1}\right) \mid \mathcal{J}_{t-1}\right]=\phi_{t}\left(\mathbf{Z}_{t-1}\right)
$$

so that:

$$
\mathrm{E}_{\mathrm{D}_{\mathbf{y}}}\left[\mathbf{e}_{t} \mid \mathcal{J}_{t-1}\right]=\mathrm{E}_{\mathrm{D}_{\mathbf{y}_{t}}}\left[\boldsymbol{\psi}_{t}\left(\mathbf{X}_{t-1}\right) \mid \mathcal{J}_{t-1}\right]-\phi_{t-1}\left(\mathbf{Z}_{t-1}\right)=\phi_{t}\left(\mathbf{Z}_{t-1}\right)-\phi_{t-1}\left(\mathbf{Z}_{t-1}\right) \neq \mathbf{0}
$$

Consequently, when a distribution shift occurs, $\mathbf{e}_{t}$ is not a mean innovation with respect to $\mathcal{J}_{t-1}$ when the relevant distribution $\mathrm{D}_{\mathbf{y}_{t}}$ is used. Without a crystal-ball predictor, the absence of which is precisely the fundamental forecasting problem, the future distribution will be unknown, and ex post, $\phi_{t-1}\left(\mathbf{Z}_{t-1}\right)$ will be a biased predictor. The crucial information reduction is from $D_{\mathbf{y}_{t}}$ to $\mathrm{D}_{\mathbf{y}_{t-1}}$, which would have major costs facing non-constancy, as will occur under extrinsic unpredictability.

\subsection{Three information sets}

If the universal information set $\mathcal{I}_{t-1}$ does not enable predictability, then the random variable in question is intrinsically unpredictable. That result applies equally to modeling and forecasting: nothing useful can be said beyond the unconditional distribution. Few observable economic variables are intrinsically unpredictable from $\mathcal{I}_{t-1}$, although modelers often seek an error that is, an issue addressed in section 4.3. In practice, the available information $\mathcal{J}_{t-1} \subset \mathcal{I}_{t-1}$ that investigators use facilitates some predictability when $\mathcal{J}_{t-1}=\sigma\left[\mathbf{Z}_{t-1}\right]$ denotes known 'standard economic forces' (e.g., for money demand, these would be incomes, prices, interest rates and lags thereof as in, e.g., Hendry and Ericsson, 1991). When $\mathbf{y}_{t}$ is not intrinsically unpredictable, but $\mathrm{D}_{\mathrm{y}_{t}}\left(\mathbf{y}_{t} \mid \mathcal{J}_{t-1}\right) \neq \mathrm{D}_{\mathrm{y}_{t}}\left(\mathbf{y}_{t}\right)$ because of instance or extrinsic unpredictability, nevertheless $\mathbf{y}_{T+1}$ may sometimes be predictable at $T$ using two additional information sets, denoted $\mathcal{L}_{T}$ and $\mathcal{K}_{T}$ above, that are not subsets of $\mathcal{J}_{T}$.

The possible information set $\mathcal{L}_{T}$ is one that would help predict the timing of a bad draw from a known fat-tailed distribution. The considerations involved seem close to those for predicting shifts of 
distributions discussed below, although instance unpredictability is a known unknown as the ex ante probability can be calculated. Empirically, however, instance unpredictability is usually an unknown unknown, because the relevant distribution is not known in advance, although characteristics thereof, such as having a fat tail, may be.

The third possible source of information, $\mathcal{K}_{T}$, is one that might help reduce extrinsic unpredictability. We assume that $\mathcal{L}_{t-1} \subset \mathcal{K}_{t-1}$ since timing predictability is needed to predict location shifts. Then, let the universal information set determining the DGP of $\left\{\mathbf{y}_{t}\right\}$ be $\mathcal{I}_{t-1}=\left(\mathcal{J}_{t-1}, \mathcal{K}_{t-1}, \mathcal{M}_{t-1}\right)$ where $\mathcal{K}_{t-\delta}=\sigma\left[\mathbf{W}_{t-\delta}\right]$ (say) which if known would explain some of the changes from $\mathrm{D}_{\mathrm{y}_{t-1}}(\cdot)$ to $\mathrm{D}_{\mathrm{y}_{t}}(\cdot)$ where $0 \leq \delta \leq 1$, and $\mathcal{M}_{t-1}$ is a relevant source of information that is unknown to investigators. Write the DGP of $\mathbf{y}_{t}$ as:

$$
\mathbf{y}_{t}=\mathbf{\Gamma} \mathbf{X}_{t-1}^{v}+\boldsymbol{\epsilon}_{t}
$$

where ${ }^{v}$ denotes column vectoring. Using only $\mathcal{J}_{t-1}$ entails marginalizing $\mathcal{I}_{t-1}$ with respect to $\mathcal{M}_{t-1}$ and $\mathcal{K}_{t-1}$, so $\mathcal{J}_{t-1}$ no longer characterizes the DGP of $\left\{\mathbf{y}_{t}\right\}$, only its local DGP (LDGP):

$$
\mathbf{y}_{t}=\mathbf{\Psi}_{t} \mathbf{Z}_{t-1}^{v}+\mathbf{e}_{t}
$$

When the resulting parameter $\boldsymbol{\Psi}_{t}$ is non-constant, but $\boldsymbol{\Gamma}$ is constant, then the information in $\mathcal{K}$ and $\mathcal{M}$ must 'explain' the breaks in (21). Shifts then alter the 'regular relationship' (e.g., in inflation relative to its usual determinants, as occurs when wars start: see Hendry, 2001). When $\left(\mathcal{J}_{t-1}, \mathcal{K}_{t-\delta}\right)$ are both known, this leads to a different LDGP, marginalized with respect to $\mathcal{M}_{t-1}$ only. When $\mathcal{K}_{t-\delta}$ is indeed the information set that induces a constant LDGP, it must do so by accounting for shifts in $\boldsymbol{\Psi}_{t}$, so assuming the simplest linear setting to illustrate:

$$
\mathbf{y}_{t}=\mathbf{\Phi}_{1} \mathbf{Z}_{t-1}^{v}+\mathbf{\Phi}_{2} \mathbf{W}_{t-\delta}^{v}+\mathbf{v}_{t}
$$

Here, $\mathbf{W}_{t-\delta}^{v}$ is dated almost contemporaneously with $\mathbf{\Psi}_{t}$ (and therefore $\mathbf{y}_{t}$ ) to produce a constant relationship, so an investigator may still need to forecast shifts in $\mathbf{W}_{t-\delta}$ at time $t$ (hence very high frequency information may help). Thus, $\mathbf{W}_{t-1}$ may not help in forecasting even if $\mathbf{W}_{t}$ would capture the shift in the relationship between $t-1$ and $t$ due to changes in (inter alia) legislation, financial innovation, technology, and policy regimes. Castle, Fawcett and Hendry (2011) discuss a variety of information sets that may improve the predictability of shifts, including leading indicators and survey data, disaggregation over time and variables, Google Trends (see Choi and Varian, 2012) and prediction markets data (like the 
Iowa electronic market), although none need help in any given instance.

When $\mathcal{M}$ accounts for the distributional shifts, such that knowing $\mathcal{J}$ and $\mathcal{K}$ does not lead to a constant LDGP, the only viable modeling approach seems to be to remove past location shifts in-sample, although that still leaves forecasting hazardous. To induce (21) from the LDGP in (22) requires a relation like:

$$
\mathbf{W}_{t}^{v}=\Pi_{t} \mathbf{Z}_{t-1}^{v}+\boldsymbol{\eta}_{t}
$$

where (23) is a projection, so does not entail an ability to forecast $\mathbf{W}_{t}$ from $\mathbf{Z}_{t-1}$ because $\boldsymbol{\Pi}_{t}$ is unknown (and perhaps unknowable) at $t-1$ : see Hendry (1988). To paraphrase Cartwright (1989), 'Breaks out (as in (21)) need breaks in (as in (23))', so it cannot be logically impossible to foresee some shifts-though it may be difficult in practice.

\subsection{Extrinsic unpredictability and economic analyses}

Due to shifts in the underlying distributions, all expectations operators must be three-way time dated, to denote the relevant random variables, the distributions being integrated over, and the available information at the time the expectations are formed, as in $\mathrm{E}_{\mathrm{D}_{\epsilon}}\left[\boldsymbol{\epsilon}_{t+1} \mid \mathcal{I}_{t-1}\right]$, which denotes the conditional expectation of $\epsilon_{t+1}$ formed at time $t$ given the information set $\mathcal{I}_{t-1}$.

\section{Definition 5}

$$
\mathrm{E}_{\mathrm{D}_{\epsilon_{t}}}\left[\epsilon_{t+1} \mid \mathcal{I}_{t-1}\right]=\int \epsilon_{t+1} \mathrm{D}_{\epsilon_{t}}\left(\epsilon_{t+1} \mid \mathcal{I}_{t-1}\right) d \epsilon_{t+1}
$$

The formulation in (24) allows for a random variable being unpredictable in mean or variance because its conditional distribution shifts in unanticipated ways relative to the conditioning information, which is perhaps the most relevant state of nature for economics.

\subsubsection{Conditional expectations}

Conditional expectations are often proved to be unbiased by arguments like the following. Let:

$$
y_{t+1}=\mathrm{E}\left[y_{t+1} \mid \mathcal{I}_{t}\right]+v_{t+1}
$$

Then taking conditional expectations of both sides:

$$
\mathrm{E}\left[v_{t+1} \mid \mathcal{I}_{t}\right]=0
$$


This result can be misinterpreted as showing that the expectation in (25) is unbiased for $y_{t+1}$, but the analysis suffers from essentially the same difficulty as the apparent paradox discussed above. Formally:

Theorem 6 When $\boldsymbol{\epsilon}_{t} \sim \mathrm{IN}_{n}\left[\boldsymbol{\mu}_{t}, \boldsymbol{\Omega}_{\boldsymbol{\epsilon}}\right]$ is extrinsically unpredictable, because future changes in $\boldsymbol{\mu}_{t}$ cannot be established in advance, the conditional expectation $\mathrm{E}_{\mathrm{D}_{\epsilon}}\left[\epsilon_{t+1} \mid \mathcal{I}_{t-1}\right]$ need not be unbiased for the outcome at $t+1$.

Proof. From (24):

$$
\mathrm{E}_{\mathrm{D}_{\epsilon}}\left[\boldsymbol{\epsilon}_{t+1} \mid \mathcal{I}_{t-1}\right]=\int \boldsymbol{\epsilon}_{t+1} \mathrm{D}_{\boldsymbol{\epsilon}_{t}}\left(\boldsymbol{\epsilon}_{t+1} \mid \mathcal{I}_{t-1}\right) \mathrm{d} \boldsymbol{\epsilon}_{t+1}=\int \boldsymbol{\epsilon}_{t+1} \mathrm{D}_{\boldsymbol{\epsilon}_{t}}\left(\boldsymbol{\epsilon}_{t+1}\right) \mathrm{d} \boldsymbol{\epsilon}_{t+1}=\boldsymbol{\mu}_{t}
$$

whereas:

$$
\mathrm{E}_{\mathrm{D}_{\epsilon_{t+1}}}\left[\epsilon_{t+1} \mid \mathcal{I}_{t-1}\right]=\int \epsilon_{t+1} \mathrm{D}_{\epsilon_{t+1}}\left(\epsilon_{t+1} \mid \mathcal{I}_{t-1}\right) \mathrm{d} \epsilon_{t+1}=\int \epsilon_{t+1} \mathrm{D}_{\epsilon_{t+1}}\left(\epsilon_{t+1}\right) \mathrm{d} \epsilon_{t+1}=\boldsymbol{\mu}_{t+1}
$$

so that $\mathrm{E}_{\mathrm{D}_{\epsilon_{t}}}\left[\epsilon_{t+1} \mid \mathcal{I}_{t-1}\right]$ does not correctly predict $\boldsymbol{\mu}_{t+1}$.

Thus, the conditional expectation $\mathrm{E}_{\mathrm{D}_{\epsilon}}\left[\boldsymbol{\epsilon}_{t+1} \mid \mathcal{I}_{t-1}\right]$ formed at $t$ is not an unbiased predictor of the outcome $\boldsymbol{\mu}_{t+1}$ at $t+1$, although the 'crystal-ball' predictor $\mathrm{E}_{\mathrm{D}_{\epsilon_{t+1}}}\left[\boldsymbol{\epsilon}_{t+1} \mid \mathcal{I}_{t-1}\right]$ remains unbiased.

Returning to (25) at time $t$ and subscripting the expectations operator as in (24):

$$
y_{t+1}=\mathrm{E}_{\mathrm{D}_{y_{t}}}\left[y_{t+1} \mid \mathcal{I}_{t}\right]+v_{t+1}
$$

so (26) becomes:

$$
\mathrm{E}_{\mathrm{D}_{y_{t}}}\left[v_{t+1} \mid \mathcal{I}_{t}\right]=0
$$

which does not entail that:

$$
\mathrm{E}_{\mathrm{D}_{y_{t+1}}}\left[v_{t+1} \mid \mathcal{I}_{t}\right]=0
$$

whereas (31) is required for an unbiased prediction. Only (29) is available to economic agents or forecasters, and after a location shift, the resulting bias can cause forecast failure, as in section 4.1.

It seems unlikely that economic agents are any more successful than professional economists in foreseeing when breaks will occur, or divining their type from one or two observations after they have happened. That link with forecast failure has important implications for economic theories about agents' expectations formation in a world with extrinsic unpredictability. General equilibrium theories rely heavily on ceteris paribus assumptions, especially that equilibria do not shift unexpectedly. The conditional expectation is the minimum mean-square error predictor in an intrinsically unpredictable process only 
when the distribution remains constant, and fails under extrinsic unpredictability. Thus, it would not be rational to use the conditional expectation under extrinsic unpredictability, which may lead agents to use forecasting devices that are robust after location shifts. Moreover, as we now show the law of iterated expectations does not apply inter-temporally when the distributions that enter the formulation change over time.

\subsubsection{The law of iterated expectations facing unanticipated shifts}

Theorem 7 When $\mathrm{h}(y, z)=\mathrm{f}(y \mid z) \mathrm{g}(z)=\mathrm{p}(y) \psi(z \mid y)$ is the joint distribution of $(y, z)$ and all the distributions involved are constant, the law of iterated expectations entails:

$$
\mathrm{E}_{z}\left[\mathrm{E}_{y}[y \mid z]\right]=\mathrm{E}_{y}[y]
$$

While well known, the following proof reveals the problems that will ensue when distributions shift:

Proof.

$$
\begin{aligned}
\mathrm{E}_{z}\left[\mathrm{E}_{y}[y \mid z]\right] & =\int_{\mathcal{Z}}\left(\int_{\mathcal{Y}} y \mathrm{f}(y \mid z) \mathrm{d} y\right) \mathrm{g}(z) \mathrm{d} z=\int_{\mathcal{Z}} \int_{\mathcal{Y}} y \mathrm{f}(y \mid z) \mathrm{g}(z) \mathrm{d} z \mathrm{~d} y \\
& =\int_{\mathcal{Y}} y\left(\int_{\mathcal{Z}} \mathrm{h}(y, z) \mathrm{d} z\right) \mathrm{d} y=\int_{\mathcal{Y}} y \mathrm{p}(y) \mathrm{d} y=\mathrm{E}_{y}[y]
\end{aligned}
$$

where $\int_{\mathcal{Z}} \mathrm{h}(y, z) \mathrm{d} z=\mathrm{p}(y)$.

When the variables correspond to a common set at different dates drawn from the same distribution, then (32) becomes:

$$
\mathrm{E}_{y_{t}}\left[\mathrm{E}_{y_{t+1}}\left[y_{t+1} \mid y_{t}\right]\right]=\mathrm{E}_{y_{t+1}}\left[y_{t+1}\right] .
$$

The formal derivation is close to that in (32), namely:

\section{Proof.}

$$
\begin{aligned}
\mathrm{E}_{y_{t}}\left[\mathrm{E}_{y_{t+1}}\left[y_{t+1} \mid y_{t}\right]\right] & =\int_{y_{t}}\left(\int_{y_{t+1}} y_{t+1} \mathrm{f}\left(y_{t+1} \mid y_{t}\right) \mathrm{d} y_{t+1}\right) \mathrm{p}\left(y_{t}\right) \mathrm{d} y_{t} \\
& =\int_{y_{t}} \int_{y_{t+1}} y_{t+1} \mathrm{f}\left(y_{t+1} \mid y_{t}\right) \mathrm{p}\left(y_{t}\right) \mathrm{d} y_{t} \mathrm{~d} y_{t+1} \\
& =\int_{y_{t+1}} y_{t+1}\left(\int_{y_{t}} \mathrm{~h}\left(y_{t+1}, y_{t}\right) \mathrm{d} y_{t}\right) \mathrm{d} y_{t+1} \\
& =\int_{y_{t+1}} y_{t+1} \mathrm{p}\left(y_{t+1}\right) \mathrm{d} y_{t+1}=\mathrm{E}_{y_{t+1}}\left[y_{t+1}\right]
\end{aligned}
$$


Thus, if the distributions remain constant, the law of iterated expectations holds.

Theorem 8 The law of iterated expectations need not hold when distributions shift:

$$
\mathrm{E}_{y_{t}}\left[\mathrm{E}_{y_{t+1}}\left[y_{t+1} \mid y_{t}\right]\right] \neq \mathrm{E}_{y_{t+1}}\left[y_{t+1}\right]
$$

\section{Proof.}

$$
\begin{aligned}
\mathrm{E}_{y_{t}}\left[\mathrm{E}_{y_{t+1}}\left[y_{t+1} \mid y_{t}\right]\right] & =\int_{y_{t}}\left(\int_{y_{t+1}} y_{t+1} \mathrm{f}_{y_{t+1}}\left(y_{t+1} \mid y_{t}\right) \mathrm{d} y_{t+1}\right) \mathrm{p}_{y_{t}}\left(y_{t}\right) \mathrm{d} y_{t} \\
& =\int_{y_{t}} \int_{y_{t+1}} y_{t+1} \mathrm{f}_{y_{t+1}}\left(y_{t+1} \mid y_{t}\right) \mathrm{p}_{y_{t}}\left(y_{t}\right) \mathrm{d} y_{t} \mathrm{~d} y_{t+1} \\
& =\int_{y_{t+1}} y_{t+1}\left(\int_{y_{t}} \mathrm{f}_{y_{t+1}}\left(y_{t+1} \mid y_{t}\right) \mathrm{p}_{y_{t}}\left(y_{t}\right) \mathrm{d} y_{t}\right) \mathrm{d} y_{t+1} \\
& \neq \int_{y_{t+1}} y_{t+1} \mathrm{p}_{y_{t+1}}\left(y_{t+1}\right) \mathrm{d} y_{t+1}=\mathrm{E}_{y_{t+1}}\left[y_{t+1}\right]
\end{aligned}
$$

as $\mathrm{f}_{y_{t+1}}\left(y_{t+1} \mid y_{t}\right) \mathrm{p}_{y_{t}}\left(y_{t}\right) \neq \mathrm{f}_{y_{t+1}}\left(y_{t+1} \mid y_{t}\right) \mathrm{p}_{y_{t+1}}\left(y_{t}\right)$ unlike the situation in (33) where there is no shift in distribution.

There are two sources of updating from, say, $\mathrm{E}_{y_{t}}\left[y_{t+1} \mid y_{t-1}\right]$ to $\mathrm{E}_{y_{t+1}}\left[y_{t+1} \mid y_{t}\right]$ : new information is embodied in $y_{t-1}$ becoming $y_{t}$; and shifts in the distribution are implied by a change from $\mathrm{E}_{y_{t}}$ to $\mathrm{E}_{y_{t+1}}$. Much of the economics literature (see e.g., Campbell and Shiller, 1987) assumes that the former is an unanticipated change, written as $\mathrm{E}\left[y_{t+1} \mid y_{t}\right]-\mathrm{E}\left[y_{t+1} \mid y_{t-1}\right]$, being an innovation, $\nu_{t}$, as the relevant information becomes known only one period later. However, that need not be not true of the latter, when the new distributional form has to be learned over time-and may have shifted again in the meantime. Even if the distribution, denoted $\mathrm{f}_{t+1}\left(y_{t+1} \mid y_{t}\right)$, became known one period later:

$$
\begin{aligned}
\mathrm{E}_{y_{t+1}}\left[y_{t+1} \mid y_{t}\right]-\mathrm{E}_{y_{t}}\left[y_{t+1} \mid y_{t-1}\right] & =\mathrm{E}_{y_{t+1}}\left[y_{t+1} \mid y_{t}\right]-\mathrm{E}_{y_{t+1}}\left[y_{t+1} \mid y_{t-1}\right] \\
& +\left(\mathrm{E}_{y_{t+1}}\left[y_{t+1} \mid y_{t-1}\right]-\mathrm{E}_{y_{t}}\left[y_{t+1} \mid y_{t-1}\right]\right) \\
& =\nu_{t}+\int y_{t+1} \mathrm{f}_{t+1}\left(y_{t+1} \mid y_{t-1}\right) \mathrm{d} y_{t+1}-\int y_{t+1} \mathrm{f}_{t}\left(y_{t+1} \mid y_{t-1}\right) \mathrm{d} y_{t} \\
& =\nu_{t}+\left(\mu_{t+1}-\mu_{t}\right)
\end{aligned}
$$

In practice, both means need to be estimated, a nearly intractable task for agents-or statisticians and econometricians-when distributions are shifting. 
The derivation deducing a martingale difference sequence (MDS) from 'no arbitrage' in, e.g., Jensen and Nielsen (1996), also explicitly requires no shifts in the underlying probability distributions. Once that is assumed, a MDS allows deducing the intrinsic unpredictability of equity price changes and hence market (informational) efficiency. Unanticipated shifts also imply unpredictability, but need not entail efficiency: informational efficiency does not follow from unpredictability per se, when the source is extrinsic rather than intrinsic. Distributional shifts occur in financial markets, as illustrated by the changing market-implied probability distributions of the S\&P500 in the Bank of England Financial Stability Report, June 2010.

In other arenas, location shifts can play a positive role in clarifying both causality, as demonstrated in White and Kennedy (2009), and super exogeneity (see Hendry and Santos, 2010). Also White (2006) considers estimating the effects of natural experiments, many of which involve large location shifts. Thus, while more general theories of the behavior of economic agents and their methods of expectations formation are required under extrinsic unpredictability, and forecasting becomes prone to failure, large shifts could also help reveal the linkages between variables.

\section{Consequences for empirical applications}

The main empirical arenas on which instance and extrinsic unpredictability impact are forecasting and modeling, so we consider these in turn.

\subsection{Instance and extrinsic unpredictability in forecasting}

Although no information could make the timing and magnitude of genuinely independent large draws predictable, once a shift has occurred, appropriate indicator variables can remove the problem from a model, even when the 'causes' are unknown. If the causes are known, the relevant variable(s) can be added. Nevertheless, knowing and adding such variables need not help in forecasting, unless one can also forecast future shifts in them. For example, oil price changes can have an important impact on inflation, and are often significant in empirical models of price inflation, but remain at least as difficult to forecast as the inflation change itself. Thus, both instance and extrinsic unpredictability may be susceptible to $e x$ post modeling, yet not improve ex ante forecasting when future shifts cannot be accurately forecast.

When $\boldsymbol{\psi}_{T+1}(\cdot)$ in (9) changes with $\mathcal{K}_{T+1}$, and thereby induces location shifts, we can write that dependence as:

$$
\mathrm{E}_{\mathrm{D}_{\mathbf{y}_{T+1}}}\left[\mathbf{y}_{T+1} \mid \mathcal{J}_{T}, \mathcal{K}_{T+1}\right]=\boldsymbol{\psi}_{0}\left(\mathbf{Z}_{T}\right)+\boldsymbol{\psi}_{1}\left(\mathbf{W}_{T+1}\right)
$$


where $\psi_{0}(\cdot)$ is constant. The second term is zero, except for shifts, accounted for by step functions or non-linear responses. At $T+1-\delta$, however, an investigator at best knows $\mathbf{Z}_{T}, \mathbf{W}_{T+1-\delta}$ and $\mathbf{D}_{\mathbf{y}_{T+1-\delta}}$, in which case:

$$
\mathrm{E}_{\mathrm{D}_{\mathbf{y}_{T+1} \delta}}\left[\mathbf{y}_{T+1} \mid \mathcal{J}_{T}, \mathcal{K}_{T+1-\delta}\right]=\boldsymbol{\psi}_{0}\left(\mathbf{Z}_{T}\right)+\mathrm{E}_{\mathrm{D}_{\mathbf{y}_{T+1-\delta}}}\left[\boldsymbol{\psi}_{1}\left(\mathcal{K}_{T+1}\right) \mid \mathcal{K}_{T+1-\delta}\right]
$$

If a location shift is unpredictable, so $\mathrm{E}_{\mathrm{D}_{\mathbf{y}_{T+1} \delta}}\left[\boldsymbol{\psi}_{1}\left(\mathcal{K}_{T+1}\right) \mid \mathcal{K}_{T+1-\delta}\right]=\mathbf{0}$, there will be no perceptible difference from the information set that enters $\boldsymbol{\psi}_{T+1}\left(\mathcal{J}_{T}\right)$. The aim, therefore, must be to ascertain available information $\mathcal{K}_{T+1-\delta}$ such that $\mathrm{E}_{\mathrm{D}_{\mathbf{y}_{T+1-\delta}}}\left[\boldsymbol{\psi}_{1}\left(\mathcal{K}_{T+1}\right) \mid \mathcal{K}_{T+1-\delta}\right] \simeq \boldsymbol{\psi}_{1}\left(\mathbf{W}_{T+1}\right)$, a daunting, but not impossible task, dependent on the specifics of the problem, although it is likely that some outliers and breaks will not be anticipated, so forecasts that are robust after breaks will often be needed.

\subsection{Unpredictability and model selection}

All three forms of unpredictability impinge on econometric modeling and model selection. A process that is intrinsically unpredictable cannot be modeled better than its unconditional distribution. For economic data, the only relevant situation where that might apply is to the error processes in models, and we consider that aspect in $\S 4.3$.

The timings and magnitudes of large draws or shifts are unanticipated ex ante with instance or extrinsic unpredictability, but once they have occurred, they can be taken into account ex post and so can be modeled. Thus, although unanticipated shifts may be highly detrimental to both economic analyses and forecasting, they need not impugn empirical modeling or model selection when correctly handled. Moreover, shifts that are not modeled can be disastrous for estimation and inference. However, impulseindicator saturation (IIS: see Hendry, Johansen and Santos, 2008, and Johansen and Nielsen, 2009) can handle multiple location shifts as well as 'remove' most of the outliers from a fat-tailed distribution. IIS creates a complete set of indicator variables $\left\{1_{\{j=t\}}\right\}=1$ when $j=t$ and 0 otherwise for $j=1, \ldots, T$ given $T$ observations, then adds these $T$ impulse indicators to the set of candidate variables. Multi-path search algorithms with tight critical values can handle more candidate variables, $N$, than $T$. As implemented in automatic model selection algorithms like Autometrics (see Doornik, 2009), IIS enables jointly locating breaks with selection over variables, functional forms and lags: see Castle, Doornik and Hendry (2012). Some well-known procedures are variants of IIS, such as the Chow (1960) test (sub-sample IIS over $T-k+1$ to $T$ without selection), and recursive estimation, which is equivalent to IIS over future samples, reducing indicators one at a time. 
Castle et al. (2011) show that IIS can avoid unmodeled outliers contaminating the selection of nonlinear models for forecasting shifts and during shifts. However, care is required in interpreting empirical models with substantively important indicator variables, when the shifts they represent could recur. Future draws are likely to include either outliers or shifts, so measures of uncertainty need to reflect that possibility, as emphasized by Hendry (2001) and Pesaran, Pettenuzzo and Timmermann (2006).

\subsection{Congruent modeling for forecasting}

The result in (17) underpins general-to-specific (Gets) model selection and the related use of congruence and encompassing as a basis for econometric model selection (see e.g., Doornik and Hendry, 2009). In terms of Gets, less is learned from $\mathcal{J}_{t-1}$ than $\mathcal{I}_{t-1}$, and the variance (when it exists) of the unpredictable component is larger. In terms of encompassing (see Bontemps and Mizon, 2003, 2008, for recent results), a later investigator may discover additional information in $\mathcal{I}_{t-1}$ beyond $\mathcal{J}_{t-1}$ which explains part of a previously unpredictable error. Nevertheless, a congruent model need not outperform in forecasting even when it coincides with the in-sample DGP, clarifying a number of findings in the empirical forecasting literature. For example, Allen and Fildes (2001) among others, find no relationship between congruence, as exemplified by rigorous mis-specification testing, and subsequent forecasting performance. Since systematic forecast failure is primarily due to extrinsically unpredictable shifts in future distributions, such a finding makes sense, but raises the question: what is the role for congruent models in forecasting? There are four potential advantages of using a congruent encompassing model for forecasting despite the omnipresent possibility of unanticipated breaks.

First, such models deliver the smallest variance for the innovation error, a key determinant of forecasterror variances, especially important when breaks do not occur over the forecast horizon. However, insample dominance does not ensure the avoidance of forecast failure, and Clements and Hendry (1998) establish the robustness after location shifts of double-differenced forecasting devices, such as $\Delta \widehat{x}_{T+1 \mid T}=$ $\Delta x_{T}$, which can outperform despite their non-congruence and non-encompassing. Clements and Hendry (2001) use that analysis to explain the outcomes of forecasting competitions, where the simplicity of a model is viewed as essential for success (see e.g., Makridakis and Hibon, 2000), but argue that is due to confounding parsimony with robustness, as such competitions did not include non-parsimonious but robust models. Castle, Fawcett and Hendry (2010) highlight the forecasting advantages of transforming a non-parsimonious model to be robust after breaks, and show that it can then even outperform the highly parsimonious yet robust device $\Delta \widehat{x}_{T+1 \mid T}=\Delta x_{T}$ after a break at time $T-1$ : how a congruent encompassing in-sample model is used in the forecast period matters when there are location shifts. 
Secondly, modeling mean shifts in-sample removes one of the sources of systematic mis-forecasting, as illustrated by Hendry and Mizon (2011). All taxonomies of forecast errors show that the equilibrium mean plays a key role therein (see e.g., Clements and Hendry, 1998): shifts, mis-estimation, or mis-specification of the equilibrium mean all induce non-zero mean forecast errors. Shifts can only be avoided by either forecasting their arrival, or using a formulation that is robust after breaks, as just discussed; mis-estimation should be small in a constant well-specified model, where the free intercept represents the mean of the dependent variable; and removing in-sample location shifts and outliers reduces that source of mis-specification.

Thirdly, eliminating from a selected model irrelevant variables that might break in the forecast period avoids a further source of forecast failure. Model selection at tight significance levels can achieve that aim jointly with selecting relevant variables, non-linear functions and lags thereof, and removing in-sample location shifts and outliers by IIS. Moreover, bias corrections after selection also reduce the impact of any adventitiously significant irrelevant variables which might shift.

Fourthly, in congruent models, decisions during model selection can be based on conventional inference procedures, such as t-tests even for I(1) data: see Sims, Stock and Watson (1990). However, when unmodeled breaks occur, heteroskedastic-consistent standard errors (HCSEs), and autocorrelationconsistent (HACSEs ) generalizations thereof (see e.g., White, 1980, and Andrews, 1991) appear to be needed. Because of the unmodeled shifts, residuals will exhibit heteroskedasticity and autocorrelation, even though those features are not present in the errors. However, HCSEs and HACSEs will incorrectly attribute the underlying uncertainty to the problems for which they were respectively designed, rather than the correct source of unmodeled shifts.

A further important issue is the ratio of the largest eigenvalue of the data second-moment matrix (say $\lambda_{1}$ ) to the smallest $\left(\lambda_{n}\right)$, albeit that is not just a problem for congruent models. Castle et al. (2010) show that location shifts reduce the collinearities between variables, having the greatest impact on the smallest eigenvalues, and since mean square forecast errors (MSFEs) depend most on $\lambda_{1} / \lambda_{n}$, changes in collinearity after a break adversely increase forecast uncertainty. This effect cannot be avoided by deleting the collinear variables, nor is the problem mitigated by orthogonalizing the variables, which can transform an external break (one affecting marginal processes) to an internal one (shifting the conditional model of interest). However, eliminating low-significance variables by model selection, and rapidly updating after a shift, especially by a relatively short moving window, can both help alleviate this problem (see e.g., Phillips, 1995, and Pesaran and Timmermann, 2007, respectively). 


\subsection{Empirical application of robust forecasting}

We now illustrate that robust transforms are a feasible tool to mitigate location shifts by pseudo ex ante forecasting of Japanese exports using monthly data over 2008(7)-2011(6), when they fell unexpectedly and drastically by more than $\mathbf{7 0 \%}$ year on year. As an autoregressive model is often difficult to outperform in forecasting, we contrast that with the robust variant of the same equation obtained by differencing.

The autoregressive model selected at $1 \%$ by Autometrics over 2000(1)-2008(6) was:

$$
\begin{aligned}
& \widehat{y}_{t}=\underset{(0.091)}{0.68} y_{t-1}+\underset{(0.090)}{0.26} y_{t-3}+\underset{(0.04)}{0.12} 1_{2000(2)}+\underset{(0.04)}{0.12} 1_{2002(1)} \\
& \widehat{\sigma}=0.039 \quad \chi^{2}(2)=0.65 \\
& \mathrm{~F}_{\text {het }}(4,95)=1.02 \quad \mathrm{~F}_{\mathrm{ar}}(6,92)=0.97 \quad \mathrm{~F}_{\text {reset }}(2,96)=2.1
\end{aligned}
$$

where $y_{t}$ denotes the annual change in the log of constant price Japanese exports, and $1_{200 z(x)}$ are indicators for $200 z(x)$. The selected model is in fact already almost 'robust' by having a near second unit root.
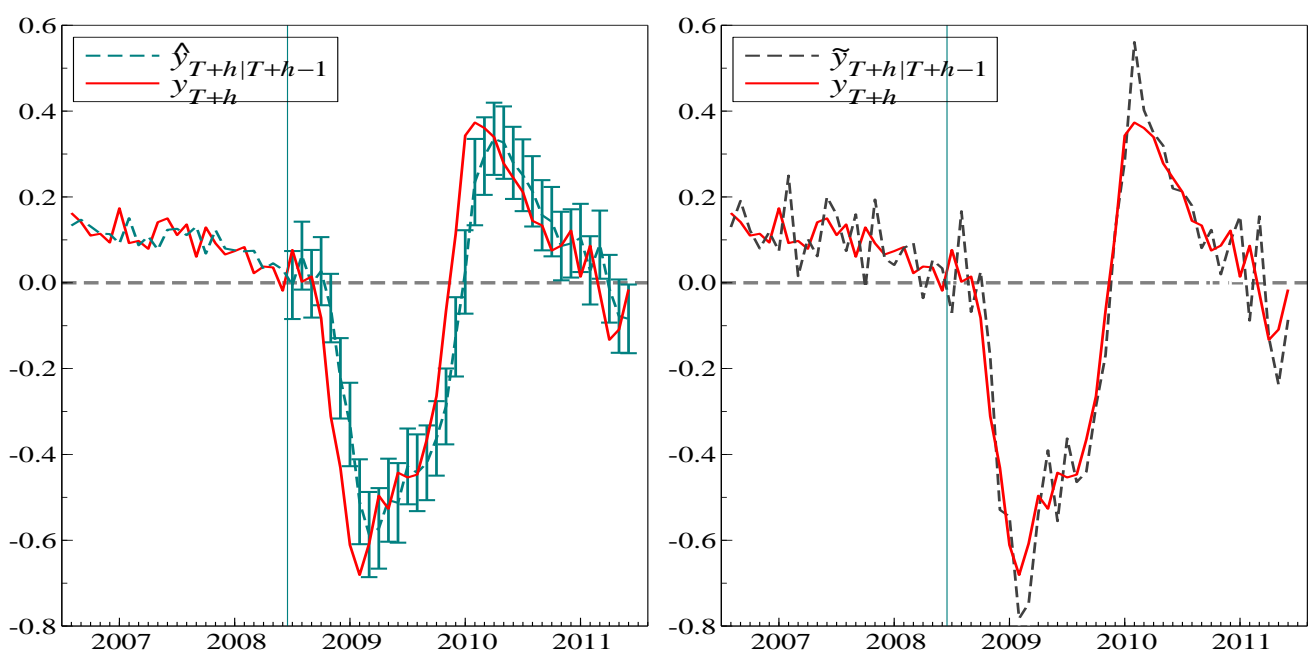

Figure 1: Forecasts of year-on-year changes in Japan's exports, 2008(7)-2011(6)

From (37), the corresponding robust device was just the simplified difference:

$$
\widetilde{y}_{t}=y_{t-1}+0.95 \Delta y_{t-1} \quad \widetilde{\sigma}=0.0755
$$




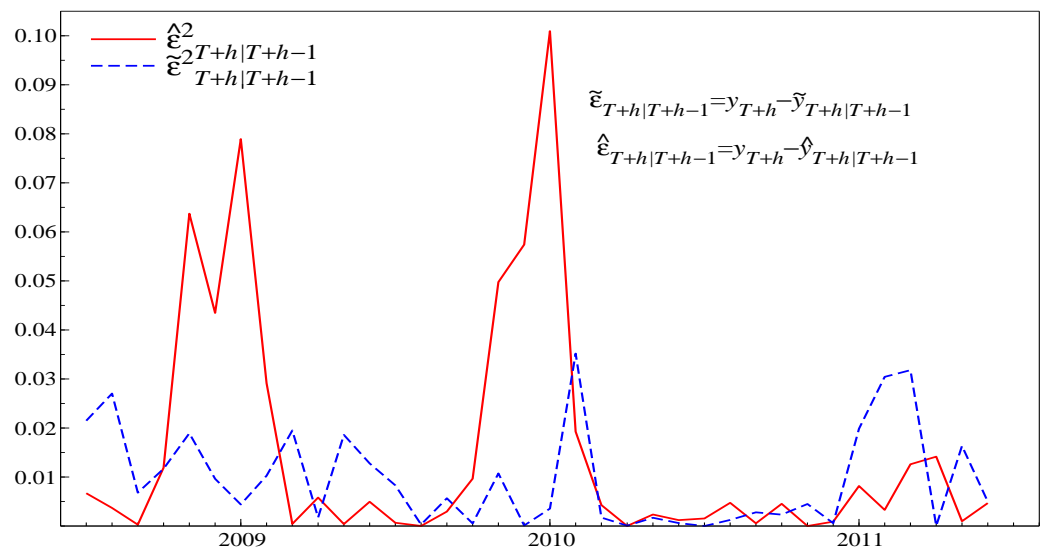

Figure 2: Squared forecast-error comparison

Their respective forecasts in Figure 1 show typical patterns. The forecasts from (37) are systematically discrepant, being above when exports fall, and below when they rise again. The error bars show $\pm 2 \widehat{\sigma}$ forecast intervals. In contrast, the robust device avoids systematic forecast failure, but overshoots at turning points plus a small 'insurance cost' when no shifts occur (no error bars are shown given the noncongruence of the device). Overall, their comparative RMSFEs are 0.124 versus 0.098 , as highlighted by Figure 2 .

Thus, while separate methods are needed to forecast a location shift (Castle et al., 2011, offer some suggestions), once such a break has occurred, robust devices can maintain forecasts on track because the DGP, and hence the data, must then incorporate the break. In addition to this example of forecasting Japanese exports demonstrating that a robust model can forecast better than an autoregression, Hendry and Mizon (2011) show that the robust version of an economic-theory based econometric model can forecast well for extensive periods despite the presence of structural change, even when the original model fails.

\section{Conclusion}

The three distinctions within unpredictability of intrinsic, instance and extrinsic, have different implications for economic analyses, econometric modeling, and forecasting. The first entails that conditioning information does not alter uncertainty, so that the unconditional distribution is the best basis for all three activities, which are therefore on an equal footing of 'uninformativeness'. The second adds the possibility that even when the distributional form is known, either unconditionally or conditionally (so 
otherwise there would be some predictability), an outcome can be highly discrepant at an unexpected time, and thereby impose substantial costs. Such outliers do not persist, but are usually in dfferenced variables (like rates of return) so can permanently change a level. The third concerns unanticipated shifts in distributions, which do persist, as with location shifts.

After they have happened, the effects from the second and third can be modeled (at a minimum by indicator variables), but if neither can be predicted on the available information, forecasts will be hazardous. Given the nature of innovation and economic behavior, it seeems highly unlikely that a metadistribution of breaks can be formulated once and for all, precluding parametrizing all possible breaks or relying on intrinsic unpredictability alone: there are too many future unknown unknowns. Nevertheless, modeling tools such as impulse-indicator saturation allow the contaminating effects of location shifts and outliers to be removed.

Important implications of extrinsic unpredictability are that conditional expectations cease to be reliably unbiased and the law of interated expectations fails to hold intertemporally. Consequently, model forms that rely on such derivations will be non-structural precisely when unanticipated location shifts occur. Moreover, economic agents may adopt robust methods to avoid systematic forecast failure following shifts. Although sources of information may exist that could help with predicting outliers or shifts, it may prove difficult to benefit from these in practice. Consequently, methods which can help ascertain the likely persistence of breaks after they have occurred seem to offer the best prospect for mitigating systematic forecast failure for both economists and economic agents.

\section{References}

Allen, P. G., and Fildes, R. A. (2001). Econometric forecasting strategies and techniques. In Armstrong, J. S. (ed.), Principles of Forecasting, pp. 303-362. Boston: Kluwer Academic Publishers.

Allen, R. C. (2009). The British Industrial Revolution in Global Perspective. Cambridge: Cambridge University Press.

Andrews, D. W. K. (1991). Heteroskedasticity and autocorrelation consistent covariance matrix estimation. Econometrica, 59, 817-858.

Apostolides, A., Broadberry, S., Campbell, B., Overton, M., and van Leeuwen, B. (2008). English Gross Domestic Product, 1300-1700: Some preliminary estimates. Discussion paper, University of Warwick, Coventry.

Barndorff-Nielsen, O. E., and Shephard, N. (2004). Power and bipower variation with stochastic volatil- 
ity and jumps. Journal of financial Econometrics, 2, 1-37.

Barndorff-Nielsen, O. E., and Shephard, N. (2006). Econometrics of testing for jumps in financial economics using bipower variation. Journal of financial Econometrics, 4, 1-30.

Barrell, R. (2001). Forecasting the world economy. In Hendry, D. F., and Ericsson, N. R. (eds.), Understanding Economic Forecasts, pp. 149-169. Cambridge, Mass.: MIT Press.

Barrell, R., Dury, K., Holland, D., Pain, N., and te Velde, D. (1998). Financial market contagion and the effects of the crises in East Asia, Russia and Latin America. National Institute Economic Review, 166, 57-73.

Barro, R. J. (2009). Rare disasters, asset prices and welfare costs. American Economic Review, 99, 243-264.

Bontemps, C., and Mizon, G. E. (2003). Congruence and encompassing. In Stigum, B. P. (ed.), Econometrics and the Philosophy of Economics, pp. 354-378. Princeton: Princeton University Press.

Bontemps, C., and Mizon, G. E. (2008). Encompassing: Concepts and implementation. Oxford Bulletin of Economics and Statistics, 70, 721-750.

Campbell, J. Y., and Shiller, R. J. (1987). Cointegration and Tests of Present Value Models. Journal of Political Economy, 95(5), 1062-1088.

Cartwright, N. (1989). Nature's Capacities and their Measurement. Oxford: Clarendon Press.

Castle, J. L., Doornik, J. A., and Hendry, D. F. (2011). Evaluating automatic model selection. Journal of Time Series Econometrics, 3 (1), DOI: 10.2202/1941-1928.1097.

Castle, J. L., Doornik, J. A., and Hendry, D. F. (2012). Model selection when there are multiple breaks. Journal of Econometrics, 169, 239-246.

Castle, J. L., Fawcett, N. W. P., and Hendry, D. F. (2010). Forecasting with equilibrium-correction models during structural breaks. Journal of Econometrics, 158, 25-36.

Castle, J. L., Fawcett, N. W. P., and Hendry, D. F. (2011). Forecasting Breaks and During Breaks. In Clements, M. P., and Hendry, D. F. (eds.), Oxford Handbook of Economic Forecasting, pp. 315353. Oxford: Oxford University Press.

Castle, J. L., and Shephard, N. (eds.)(2009). The Methodology and Practice of Econometrics. Oxford: Oxford University Press.

Choi, H., and Varian, H. (2012). Predicting the present with Google Trends. Economic Record, 88, 2-9. Chow, G. C. (1960). Tests of equality between sets of coefficients in two linear regressions. Economet- 
rica, 28, 591-605.

Clements, M. P., and Hendry, D. F. (1998). Forecasting Economic Time Series. Cambridge: Cambridge University Press.

Clements, M. P., and Hendry, D. F. (2001). Explaining the results of the M3 forecasting competition. International Journal of Forecasting, 17, 550-554.

Clements, M. P., and Hendry, D. F. (2005). Information in economic forecasting. Oxford Bulletin of Economics and Statistics, 67, 713-753.

Doob, J. L. (1953). Stochastic Processes. New York: John Wiley Classics Library. 1990 edition.

Doornik, J. A. (2009). Autometrics. In Castle, and Shephard (2009), pp. 88-121.

Doornik, J. A., and Hendry, D. F. (2009). Empirical Econometric Modelling using PcGive: Volume I. London: Timberlake Consultants Press.

Granger, C. W. J., and Pesaran, M. H. (2000a). A decision-theoretic approach to forecast evaluation. In Chon, W. S., Li, W. K., and Tong, H. (eds.), Statistics and Finance: An Interface, pp. 261-278. London: Imperial College Press.

Granger, C. W. J., and Pesaran, M. H. (2000b). Economic and statistical measures of forecasting accuracy. Journal of Forecasting, 19, 537-560.

Hendry, D. F. (1988). The encompassing implications of feedback versus feedforward mechanisms in econometrics. Oxford Economic Papers, 40, 132-149.

Hendry, D. F. (2001). Modelling UK inflation, 1875-1991. Journal of Applied Econometrics, 16, 255275.

Hendry, D. F., and Ericsson, N. R. (1991). Modeling the demand for narrow money in the United Kingdom and the United States. European Economic Review, 35, 833-886.

Hendry, D. F., Johansen, S., and Santos, C. (2008). Automatic selection of indicators in a fully saturated regression. Computational Statistics, 33, 317-335. Erratum, 337-339.

Hendry, D. F., and Massmann, M. (2007). Co-breaking: Recent advances and a synopsis of the literature. Journal of Business and Economic Statistics, 25, 33-51.

Hendry, D. F., and Mizon, G. E. (2011). Econometric modelling of time series with outlying observations. Journal of Time Series Econometrics, 3 (1), DOI: 10.2202/1941-1928.1100.

Hendry, D. F., and Santos, C. (2010). An automatic test of super exogeneity. In Watson, M. W., Bollerslev, T., and Russell, J. (eds.), Volatility and Time Series Econometrics, pp. 164-193. Oxford: 


\section{Oxford University Press.}

Jensen, B. A., and Nielsen, J. A. (1996). Pricing by no arbitrage. In Cox, D. R., Hinkley, D. V., and Barndorff-Nielsen, O. E. (eds.), Time Series Models: In Econometrics, Finance and other Fields, pp. 179-225. London: Chapman and Hall.

Johansen, S., and Nielsen, B. (2009). An analysis of the indicator saturation estimator as a robust regression estimator. In Castle, and Shephard (2009), pp. 1-36.

Leitch, G., and Tanner, J. E. (1991). Economic forecast evaluation: Profits versus the conventional error measures. American Economic Review, 81, 580-590.

Makridakis, S., and Hibon, M. (2000). The M3-competition: Results, conclusions and implications. International Journal of Forecasting, 16, 451-476.

Pesaran, M. H., Pettenuzzo, D., and Timmermann, A. (2006). Forecasting time series subject to multiple structural breaks. Review of Economic Studies, 73, 1057-1084.

Pesaran, M. H., and Timmermann, A. (2007). Selection of estimation window in the presence of breaks. Journal of Econometrics, 137, 134-161.

Phillips, P. C. B. (1995). Automated forecasts of Asia-Pacific economic activity. Asia-Pacific Economic Review, 1, 92-102.

Sims, C. A., Stock, J. H., and Watson, M. W. (1990). Inference in linear time series models with some unit roots. Econometrica, 58, 113-144.

Soros, G. (2008). The New Paradigm for Financial Markets. London: Perseus Books.

Soros, G. (2010). The Soros Lectures. Philadelphia: Perseus Books.

Taleb, N. N. (2007). The Black Swan. New York: Random House.

Taleb, N. N. (2009). Errors, robustness, and the fourth quadrant. International Journal of Forecasting, 25, 744-759.

White, H. (1980). A heteroskedastic-consistent covariance matrix estimator and a direct test for heteroskedasticity. Econometrica, 48, 817-838.

White, H. (2006). Time series estimation of the effects of natural experiments. Journal of Econometrics, $135,527-566$.

White, H., and Kennedy, P. (2009). Retrospective estimation of causal effects through time. In Castle, and Shephard (2009), pp. 59-87. 